\title{
Eufáusidos de Isla de Pascua
}

\author{
Oscar Robledo B. y Armando Mujica R. \\ Universidad Católica del Norte, Facultad de Ciencias del Mar \\ Casilla 117, Coquimbo, Chile. \\ E-mail: amujica@nevados.cecun.ucn.cl
}

Recibido 14 agosto 1997; versión corregida 15 octubre 1999; aceptado 19 octubre 1999.

RESUMEN. Se analiza la distribución espacial y abundancia relativa del Orden Euphausiacea en aguas circundantes a Isla de Pascua en mayo de 1994. Las muestras zooplanctónicas se obtuvieron en 16 estaciones oceanográficas mediante pescas oblicuas efectuadas con redes Bongo de $330 \mu$, entre la superficie y $250 \mathrm{~m}$ de profundidad. Se analiza la estructura comunitaria de los eufáusidos, mediante índices de constancia y dominancia.

En todas las estaciones se registró la presencia de eufáusidos, encontrándose las mayores diversidades en las estaciones más alejadas de la isla. Stylocheiron suhmii y S. affine fueron las especies más abundantes y frecuentes.

La posible influencia que pudiese tener la isla y las condiciones oceanográficas en la agregación de organismos zooplanctónicos, no pudo ser detectada en la distribución y abundancia de las especies de eufáusidos, debido principalmente a la escala espacial del muestreo. Se sugiere que el comportamiento y migración vertical de las especies, puede ser un factor determinante en la estructura comunitaria de los eufáusidos en torno a este ecosistema insular.

Palabras claves: Distribución, abundancia, eufáusidos, Isla de Pascua.

\section{Euphausiids of Easter Island}

\begin{abstract}
The spatial distribution and relative abundance of Euphausiacea, during May 1994, in waters around Easter Island, are analyzed. Zooplankton was sampled at 16 oceanographic stations. Double oblique sampling was done with Bongo net between the surface and $250 \mathrm{~m}$ depth. The community structure of euphausiids was analyzed using constancy and dominance.

Euphausiids were present at all stations, and its diversity was most high in the most oceanic stations. Considering the numeric constancy and dominance the most important species were Stylocheiron affine and S. suhmii.

The island presence and oceanographic influence in spatial distribution and relative abundance patterns of euphausiids around island don't was detected. It was associated to sampling scale. Probably the spatial distribution and relative abundance of euphausiids is related principally to behavior and vertical migration.
\end{abstract}

Key words: Distribution, abundance, euphausiids, Easter Island.

\section{INTRODUCCIÓN}

El Orden Euphausiacea es un importante componente del zooplancton, debido a su amplia distribución y abundancia en diferentes regiones oceánicas, como también por su rol en la transferencia energética de los niveles primarios de la trama trófica del ecosistema pelágico (Ponomareva, 1963; Mauchline y Fisher, 1969; Antezana y Brinton, 1981; Kinsey y Hopkins, 1994).

Las principales variables ambientales relacionadas con la distribución espacial y abundancia relativa de los eufáusidos, son la temperatura y salinidad, que afectan principalmente los patrones de distribución horizontal y vertical de las especies (Antezana y Cornejo, 1979; Brinton, 1979; Lavaniegos-Espejo y Lara, 1989). En los márgenes continentales, se ha establecido que procesos oceanográficos, como surgencia y circulación, determinan la permanencia espacio-temporal y estructura de las poblaciones de eufáusidos (Barange y Pillar, 1992; Gibbons et al., 1995). Así también, otros factores ambientales, como la concentración de oxígeno disuelto y nutrientes, han sido utilizados para describir la distribución y migración vertical de algunas especies (Antezana, 1978; Brinton, 1979). A pesar de estas referencias, existe escasa información referida al comportamiento de eufáusidos en aguas circundantes a islas. 
Los ecosistemas insulares representan normalmente perturbaciones en los sistemas de flujo oceánico, determinando variaciones en la biomasa y la producción biológica en sus inmediaciones, debido principalmente a procesos advectivos (Simpson y Tett, 1986; Boehlert et al., 1992). La dinámica de las aguas circundantes a islas está determinada por la velocidad y dirección del flujo oceánico, la posición geográfica, tamaño y forma de la isla, así como la topografía de su relieve submarino (Boehlert y Genin, 1987; Heywood et al., 1992).

La mayor cantidad de información referente al comportamiento del zooplancton en torno a islas, se ha obtenido del análisis del meroplancton, especialmente huevos y larvas de peces (Rojas, 1980). Esto debido a que se ha tratado de contribuir al conocimiento de los mecanismos de permanencia espacio-temporal de poblaciones insulares endémicas (Leis y Miller, 1976; Boehlert y Mundy, 1993). Sin embargo, la abundancia relativa del meroplancton es baja con relación al resto de los zooplancteres y depende principalmente de los ciclos reproductivos de las especies, siendo abundantes en determinadas épocas del año (Omori e Ikeda, 1984). Por tal razón, los grupos holoplanctónicos pueden ser mejores descriptores de las condiciones oceanográficas de las aguas circundantes a islas (Miller, 1974; Palma, 1985; Townsed et al., 1986).

Las investigaciones oceanográficas han sido escasas en Isla de Pascua. Los primeros estudios describieron las condiciones térmicas y salinas de esa región (Gana, 1885; Bahamonde, 1953). Posteriormente, se han descrito y caracterizado las principales masas de agua presentes a más de $100 \mathrm{~km}$ de la isla (Silva, 1992). Investigaciones posteriores, han descrito la presencia de una masa de agua subtropical en la zona epipelágica del área costera, caracterizada por altas temperatura y salinidad (Olivares y Moraga, 1993; Moraga y Olivares, 1996). Además, se ha establecido que la concentración de nutrientes y clorofila es baja, concordando con las características oligotróficas de las regiones subtropicales (Olivares y Moraga, 1993).

En relación a los estudios planctónicos, Mujica (1993), describe la presencia de copépodos, quetognatos y eufáusidos, los cuales están presentes en toda el área analizada, siendo los componentes holoplanctónicos los más importantes del zooplancton, de acuerdo a su dominancia.
En el presente trabajo se entrega información referente a la composición y distribución de abundancia de los eufáusidos en aguas circundantes a Isla de Pascua, como aporte al conocimiento de la biodiversidad de este ecosistema insular.

\section{MATERIALES Y MÉTODOS}

A bordo del B/I Vidal Gormaz, durante los días 22 y 23 de mayo de 1994 y en aguas circundantes a Isla de Pascua $\left(27^{\circ} 08^{\prime}\right.$ 'S $\left.-109^{\circ} 26^{\prime} \mathrm{W}\right)$, se tomaron muestras de zooplancton en 16 estaciones oceanográficas dispuestas en dos transectos perpendiculares a la costa, ubicados al norte y sur de la isla, que incluyeron lances diurnos y nocturnos (Fig. 1A).

Para la obtención de las muestras se utilizaron redes Bongo, de $61 \mathrm{~cm}$ de diámetro y $330 \mathrm{~mm}$ de abertura de malla, las que fueron arrastradas en forma doble oblicua, entre la superficie y $250 \mathrm{~m}$ de profundidad. El volumen de agua filtrado por la red, fue determinado mediante un flujómetro. Conjuntamente con las pescas de zooplancton se obtuvieron registros de temperatura $\left({ }^{\circ} \mathrm{C}\right)$, salinidad (psu) y nutrientes $\left(\mathrm{mmol} / \mathrm{dm}^{3}\right)$ entre la superficie y $1.500 \mathrm{~m}$ de profundidad (Moraga y Olivares, 1996).

Las muestras de zooplancton se fijaron en solución de formaldehído al 5\% en agua de mar y sólo los ejemplares adultos se identificaron a nivel específico, considerando los caracteres taxonómicos descritos por Boden y Brinton (1957), Mauchline y Fisher (1969), Ramírez (1971), Cornejo (1977), Mauchline (1980) y Antezana y Brinton (1981).

La abundancia relativa fue expresada como número de individuos en $1.000 \mathrm{~m}^{3}$ de agua de mar filtrada. Para atenuar las diferencias en la abundancia relativa en la representación gráfica, el diámetro de las esferas en las figuras, corresponde al valor de la raíz cúbica del número de ejemplares presentes en $1.000 \mathrm{~m}^{3}$ de agua filtrada.

La frecuencia de ocurrencia o constancia numérica (porcentaje entre el número de estaciones con presencia de cada especie y el número total de estaciones) y la dominancia numérica de las especies (porcentaje entre el número total de individuos de cada especie y el número total de eufáusidos capturados), fue valorada de acuerdo a la escala propuesta por Bodenheimer (1955). 

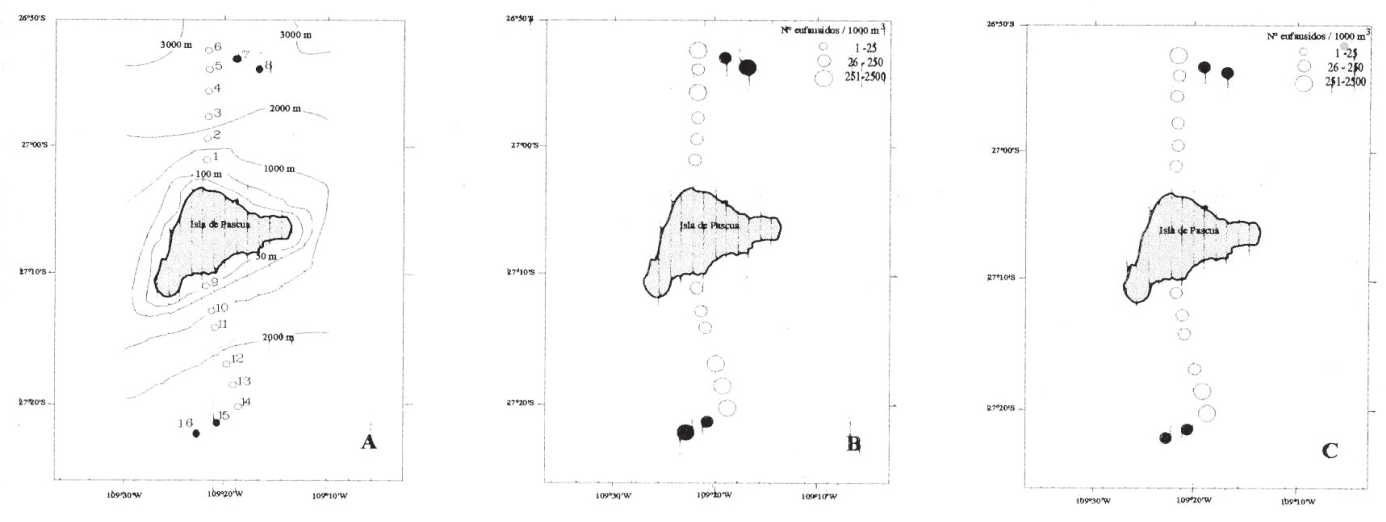

Figura 1. A: Área de estudio y ubicación de las estaciones oceanográficas. B: Distribución y abundancia relativa de los eufáusidos. C: Distribución y abundancia relativa de Stylocheiron suhmii. o estaciones diurnas, $\bullet$ estaciones nocturnas.

Figure 1. A: Area of study and positions of oceanografic stations. B: Distribution and relative abundance of euphausiids. C: Distribution and relative abundance of $S$. suhmii. o diurnal stations, $\bullet$ nocturnal stations.

\section{RESULTADOS}

Los eufáusidos se capturaron en todas las estaciones de muestreo (100\% de constancia), registrándose una escasa abundancia relativa, que fluctuó entre 74 y 551 ind. $/ 1.000 \mathrm{~m}^{3}$. Al norte de la isla, las mayores abundancias se encontraron en las estaciones más distantes de la costa (Fig. 1B), con máximos de 403 y 551 ind. $/ 1.000 \mathrm{~m}^{3}$ en las estaciones 6 y 8 , respectivamente. La menor abundancia se determinó en la estación más costera, al sur de la isla (74 ind./1.000 m³ , Est.9). En general, al sur de la isla la abundancia aumentó progresivamente hacia mar afuera (Fig. 1B).

Se identificaron seis especies pertenecientes a tres géneros de la familia Euphausiidae: Stylocheiron suhmii, S. affine, S. carinatum, S. abbreviatum, Euphausia brevis y Thysanopoda aequalis. Todas las cuales han sido registradas para la región central del Pacífico Sur (Brinton, 1962; Antezana, 1970; 1978), siendo esta la primera vez que son citadas alrededor de Isla de Pascua.

De ellas, S. suhmii y S. affine presentaron la distribución espacial más amplia y las mayores abun- dancias relativas (Fig. 1C y 2A). Ambas con valores de constancia superiores al $90 \%$ y con las mayores dominancias (Tabla I). Las mayores abundancias de estas especies fueron de 313 y 141 ind./1.000 $\mathrm{m}^{3}$, en las estaciones 14 y 16 , respectivamente. La distribución de $S$. suhmii fue similar a la encontrada para el total de los eufáusidos capturados y fue la única capturada en la estación más próxima al sur de la isla (Est. 9).

S. affine se encontró en 15 de las 16 estaciones y su abundancia, relativamente homogénea, fluctuó entre 14 y 141 ind./1.000 $\mathrm{m}^{3}$. Sus máximos de abundancia se detectaron al sur de la isla, desde donde se observó un aumento progresivo desde la costa hacia mar afuera.

S. carinatum se encontró presente en nueve estaciones, a ambos lados de la isla y en bajas cantidades, con valores extremos de 6 y 44 ind. $/ 1.000 \mathrm{~m}^{3}$ (Fig. 2B). Sus mayores abundancias se determinaron en ambos transectos, en las estaciones más alejadas de la costa, especialmente durante la noche.

S. abbreviatum se capturó sólo en cinco estacio- 

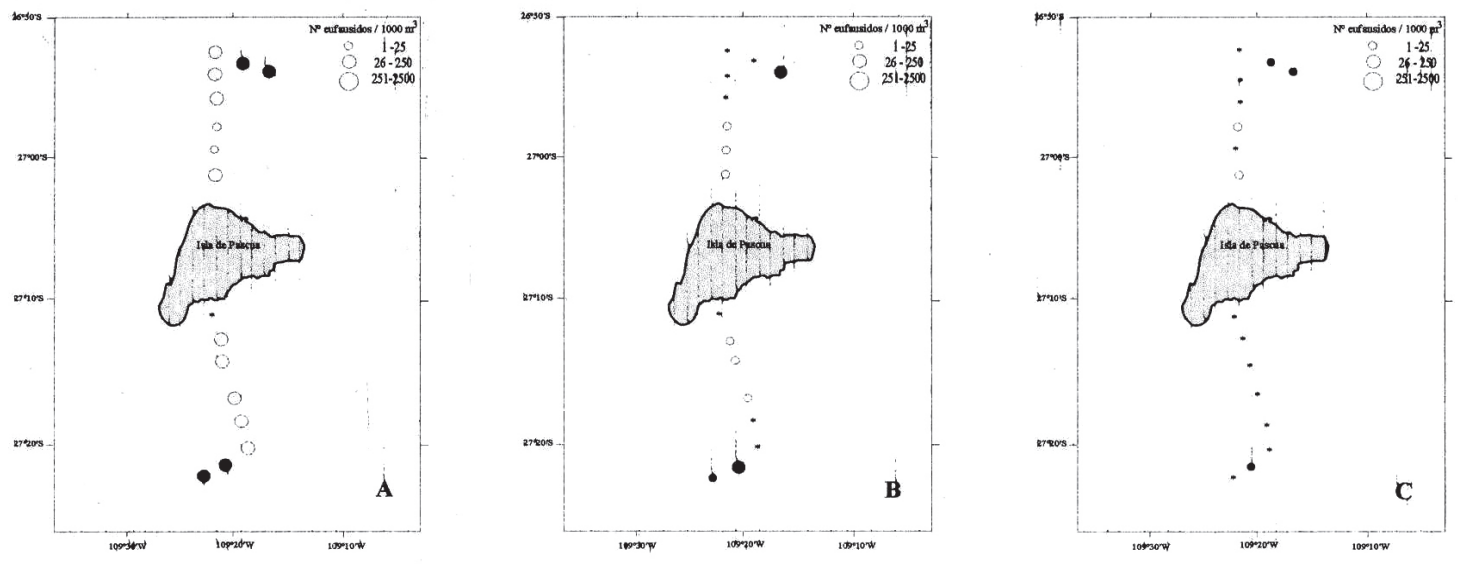

Figura 2. Distribución y abundancia relativa de A: Stylocheiron affine. B: S. carinatum. C: S. abbreviatum. o estaciones diurnas, $\bullet$ estaciones nocturnas, * estaciones sin captura.

Figure 2. Distribution and relative abundance of A: Stylocheiron affine. B: S. carinatum. C: S. abbreviatum. o diurnal stations, $\bullet$ nocturnal stations, *stations without catch.

nes, ubicadas principalmente lejos de la costa (Fig. 2C) y en pequeñas cantidades, de 7 a 25 ind./1.000 $\mathrm{m}^{3}$. Se capturó preferentemente en las estaciones nocturnas. Fue la única especie accidental por su escasa dominancia (Tabla 1).

E. brevis y $T$. aequalis fueron las únicas de frecuencia accidental y se encontraron sólo en las dos estaciones más alejadas de cada transecto, correspondiendo en ambos casos a lances nocturnos. La dominancia numérica de estas especies fue intermedia (Tabla 1), lo que en conjunto con la baja frecuencia de ocurrencia de ellas, la hora en que se efectuaron las estaciones, y el comportamiento migratorio vertical de los eufáusidos, induce a pensar que su presencia fue subestimada por el tipo de muestreo empleado.

\section{DISCUSIÓN}

La baja abundancia relativa de los eufáusidos alrededor de Isla de Pascua es coincidente con lo señalado por Mauchline y Fisher (1969) para otras regiones subtropicales y tropicales del Océano Pacífico, aun teniendo en consideración lo limitado en

Tabla 1. Abundancia relativa, constancia y dominancia numérica de especies de eufáusidos.

Table 1. Relative abundance, constance and numeric dominance of the euphusiids species.

\begin{tabular}{|lccc|}
\hline Especie & $\begin{array}{c}\text { Abundancia relativa } \\
\left(\mathbf{N} / \mathbf{1 . 0 0 0} \mathbf{~ m}^{\mathbf{3}}\right)\end{array}$ & $\begin{array}{c}\text { Constancia } \\
(\mathbf{\%})\end{array}$ & $\begin{array}{c}\text { Dominancia } \\
(\%)\end{array}$ \\
\hline S. suhmii & 2.455 & 100 & 62,3 \\
S. affine & 1.015 & 93,8 & 25,7 \\
S. carinatum & 161 & 56,3 & 4,1 \\
S. abbreviatum & 66 & 31,3 & 1,7 \\
E. brevis & 132 & 12,5 & 3,3 \\
T. aequalis & 113 & 12,5 & 2,9 \\
\end{tabular}


tiempo y espacio del muestreo efectuado, además del hecho que el muestreo se efectuó a diferentes horas, en las que la capturabilidad, accesibilidad y vulnerabilidad de las especies planctónicas es variada.

S. suhmii fue la especie que por su frecuencia y dominancia caracterizó la comunidad de eufáusidos en torno a la isla. Al respecto, Brinton (1962), establece que $S$. suhmii es una especie dominante entre los eufáusidos de la región subtropical del Pacífico Sur, región donde se encuentra la Isla de Pascua. Según Mauchline (1980), la región subtropical podría constituir uno de los centros de dispersión de esta especie hacia otras zonas del giro anticiclónico del Pacífico Sur. Fue la única especie capturada en la estación más próxima a la costa del lado sur de la isla, que es la mas protegida, la de menor profundidad y en la que se encontró la menor temperatura (Moraga y Olivares, 1996).

S. affine, la otra especie de alta frecuencia y dominancia, ha sido señalada como uno de los principales componentes de los centros de los giros oceánicos del Pacífico norte y sur. Esta especie ha sido calificada como cosmopolita, dado que su distribución espacial comprende regiones oceánicas muy diversas, caracterizadas por la presencia de aguas cálidas o templadas (Brinton, 1979; Mauchline y Fisher, 1969; Mauchline, 1980).

S. abbreviatum que fue la especie más escasa y se encontró en cantidades relativamente menores a las descritas por Brinton (1962) en el centro del Pacífico sur (1 a 50 ind./1.000 m³). La escasa cantidad de individuos, ha sido descrita para otras regiones subtropicales, como el Atlántico norte (Kinsey y Hopkins, 1994).

En el Océano Pacífico, esta especie se ha descrito para sectores ubicados en la región ecuatorial y subtropical del Pacífico norte, áreas donde se han encontrado en mayor abundancia entre 50 y 500 ind./ $1.000 \mathrm{~m}^{3}$ (Brinton, 1962).

Las estaciones más distantes efectuadas en ambos lados de la isla (Estaciones 8 y 16), presentaron la mayor riqueza de especies, difiriendo entre ellas en una especie (S. abbreviatum), y son las únicas en que se encontró E. brevis y T. aequalis. Su presencia podría relacionarse con el patrón de migración vertical de ellas, puesto que esas estaciones fueron las únicas muestreadas durante la noche. Estas observaciones concuerdan con los resultados obtenidos por Kinsey y Hopkins (1994), en las regiones subtropicales del Atlántico Norte. Estos autores se- ñalan que el comportamiento migratorio vertical de E. americana, E. brevis y E. tenera contribuye en forma importante a la estructura de las poblaciones de eufáusidos en los estratos superficiales de la columna de agua durante la noche, representando más del $50 \%$ de todas las especies capturadas. Este comportamiento fue descrito por Mauchline y Fisher (1969) y Mauchline (1980), quienes señalan que el rango de distribución vertical durante el día varía entre 200-400 m de profundidad, alcanzando los 50 $\mathrm{m}$ durante la noche, lo que implica migraciones promedio superiores a $300 \mathrm{~m}$.

La baja dominancia de E. brevis (<75 ind./1.000 $\mathrm{m}^{3}$ ), coincide con los resultados descritos en otras regiones oceánicas. En efecto, Baker (1970) en aguas próximas a las Islas Canarias, encontró promedios de $54 \mathrm{ind} / 1.000 \mathrm{~m}^{3}$ en pescas efectuadas entre $200 \mathrm{y}$ $600 \mathrm{~m}$ de profundidad, agregando que más del $50 \%$ de los individuos se encontró entre 250 y $300 \mathrm{~m}$. Esto indicaría una evasión a la red en los estratos superficiales durante el día, pudiendo ser más eficientes las capturas nocturnas, lo que indicaría que la abundancia de esta especie fue subestimada (Mauchline, 1980; Watkins et al., 1985). Al respecto, Brinton (1962) estimó que su abundancia en la región central del Pacífico, fluctuó entre 50 y 499 ind./1.000 $\mathrm{m}^{3}$, siendo más abundante en el Pacífico sur.

Respecto de la presencia de T. aequalis, en estaciones practicadas durante la noche, se puede señalar, que las especies del género Thysanopoda presentan una activa migración vertical, distribuyéndose durante el día entre 100 y 400 m, mientras que en la noche se encuentra alrededor de los $150 \mathrm{~m}$ (Mauchline, 1980; Kinsey y Hopkins, 1994). Esto ha llevado a considerar a este género junto con Euphausia, como los migradores verticales más activos. Según Youngbluth, (1976), Euphausia se distribuye principalmente bajo los $300 \mathrm{~m}$ durante el día. En regiones subtropicales del Atlántico norte, T. aequalis se encuentra en escasa cantidad (Kinsey y Hopkins, 1994), mientras que en otras regiones no se han realizado estimaciones cuantitativas, debido a que usualmente se ha capturado un número reducido de ejemplares adultos, lo que se ha asociado a su elevada capacidad natatoria (Mauchline y Fisher, 1969; Brinton, 1979).

La escasa abundancia de $S$. carinatum fue menor a la estimada para la región central del Pacífico por Brinton (1962). Al respecto, este autor señala que las mayores densidades se han encontrado en la 
región oeste de los centros del giro oceánico del Pacífico, con densidades entre 50 y 500 ind./1.000 $\mathrm{m}^{3}$. Por su abundancia relativa en la región subtropical del Atlántico norte, esta especie ha sido clasificada como la segunda en importancia numérica por Kinsey y Hopkins (1994).

La mayor riqueza de especies registrada en las últimas estaciones efectuadas a ambos lados de la isla, debe ser considerada con cautela, puesto que en ambos casos las pescas se hicieron en horas de obscuridad. Por lo tanto, su composición específica puede estar influenciada por la distribución batimétrica de las especies y la migración vertical que éstas realizan.

En general, el mayor número de especies encontradas en las estaciones más distantes de la isla, es coincidente con las altas diversidades descritas para las regiones oceánicas subtropicales y tropicales (Brinton, 1979; Margalef, 1980; Omori e Ikeda, 1984). Esto podría ser explicado por la gran homogeneidad horizontal que presentan las condiciones físicas y químicas en los giros centrales del Pacífico norte y sur (Loeb, 1979; Silva, 1992; Olivares y Moraga, 1993).

El aumento de la riqueza específica en estaciones muestreadas en horas de obscuridad, indica la influencia de la componente temporal diaria que puede tener en la dinámica de las comunidades zooplanctónicas, y en especial, la activa migración vertical que presentan algunas especies, como $E$. brevis y T. aequalis (Mauchline y Fisher, 1969; Mauchline, 1980).

Finalmente, considerando que los centros de los giros oceánicos se caracterizan por presentar diversidades altas, niveles tróficos de baja biomasa, escasa variabilidad estacional y baja productividad primaria y secundaria (McGowan y Walker, 1985), es posible que la estructura de los eufáusidos en aguas circundantes a Isla de Pascua sea regulada principalmente por el gradiente vertical de las condiciones oceanográficas. Este podría actuar en la determinación de la estrategia trófica de las especies, que estaría determinando diferencias en los patrones de distribución vertical y dieta de los especímenes.

\section{AGRADECIMIENTOS}

Los autores agradecen al Comité Oceanográfico Nacional y a la Facultad de Ciencias del Mar de la Universidad Católica del Norte, que permitieron mediante el financiamiento e infraestructura la ejecución del presente trabajo. Al comandante y tripulación del B/I Vidal Gormaz y al equipo de científicos que colaboraron en la toma de muestras. Al Dr. Sergio Palma, por sus valiosos comentarios críticos al manuscrito.

\section{REFERENCIAS}

Antezana, T. 1970. Eufáusidos de la costa de Chile. Su rol en la economía del mar. Rev. Biol. Mar., 14 (2): 19-27.

Antezana, T. 1978. Distribution of euphausiids in the Chile-Peru Current with particular reference to the endemic Euphausia mucronata and the oxygen minima layer. Thesis Ph.D. University of California, San Diego. 489 pp.

Antezana, T. y E. Brinton. 1981. Euphausiacea. En: D. Boltovskoy (ed.). Atlas del Zooplancton del Atlántico Sudoccidental y métodos de trabajo con el zooplancton marino. INIDEP, Mar del Plata, pp. 681-698.

Antezana, T. y M. Cornejo. 1979. Distribución de los eufáusidos epipelágicos del Ecuador y su validez como indicadores de masas de agua (Crustacea, Zooplancton). Bol. Inst. Oceanogr. Ecuador, 3 (1): 1-30.

Bahamonde, N. 1953. Observaciones sobre salinidad realizadas durante el viaje a Isla de Pascua en el transporte "Angamos". Julio-Agosto de 1953. Inf. Geogr., 3/4: 76-78.

Baker, A. 1970. The vertical distribution of euphausiids near Fuerteventura, Canary Islands ('Discovery' Sond Cruise, 1965). J. Mar. Biol. Ass. U. K., 50: 301-342.

Barange, M. y S. Pillar. 1992. Cross-shelf circulation, zonation and maintenance mechanisms of Nyctiphanes capensis and Euphausia hanseni (Euphausiacea) in the northern Benguela upwelling system. Continental Shelf Res., 12 (9): 1027-1042.

Boden, B. y E. Brinton. 1957. The euphausiids crustaceans Thysanopoda aequalis Hansen y Thysanopoda subaequalis Boden, their taxonomy and distribution in the Pacific. Limnol. Oceanogr., 2: 337-341.

Bodenheimer, F. 1955. Précis d'écologie animale. Ed. Payot, París. 25 pp. 
Boehlert, G. y A. Genin. 1987. A review of the effects of seamounts on biological processes. In: Seamounts, islands and atolls. B. Keating, P. Fryer, R. Batiza and G. Boehlert Eds. Geophys. Monogr., 43: 319-334.

Boehlert, G. y B. Mundy. 1993. Ichthyoplankton assemblages at seamounts and oceanic islands. Bull. Mar. Sci., 53(2): 336-361.

Boehlert, G., W. Watson y L.C. Sun. 1992. Horizontal and vertical distributions of larval fishes around an isolated oceanic island in the tropical Pacific. Deep-Sea Res., 39(3-4): 439-466.

Brinton, E. 1962. The distribution of Pacific euphausiids. Bull. Scripps Inst. Oceanogr., 8: 51270 .

Brinton, E. 1979. Parameters relating to the distribution of planktonic organisms, especially Euphausiids in the eastern tropical Pacific. Prog. Oceanogr., 8: 125-189.

Cornejo, M. 1977. Estudio preliminar de los eufáusidos del mar ecuatoriano. Bol. Inst. Oceanogr. Ecuador, 3(1): 1-46.

Gana, I. 1885. Descripción de la Isla de Pascua. Rev. Mar., 4: 369-384.

Gibbons, M., M. Barange y L. Hutchings. 1995. Zoogeography and diversity of euphausiids around southern Africa. Mar. Biol., 123: 257-268.

Heywood, K., E. Barton y J. Simpson. 1992. The effects of flow disturbance by an oceanic island. J. Mar. Res., 48: 55-73.

Kinsey, S.T. y T.L. Hopkins. 1994. Trophic strategies of euphausiids in a low-latitude ecosystem. Mar. Biol., 118: 651-661.

Lavaniegos-Espejo, B. y J. Lara. 1989. Effects of the 1982-83 El Niño event on the euphausiid populations of the Gulf of California. CalCofi Rep., 30: 73-85.

Leis, J. y J. Miller. 1976. Offshore distributional patterns of hawaiian fish larvae. Mar. Biol., 36: 359-367.

Loeb, V. 1979. Larval fishes in the zooplankton community of the north Pacific central gyre. Mar. Biol., 53: 173-191.

Margalef, R. 1980. Ecología. Omega, Barcelona, 937 pp.
Mauchline, J. 1980. The biology of mysids and euphausiids. Adv. Mar. Biol., 18: 373-681.

Mauchline, J. y L. Fisher. 1969. The biology of euphausiids. Adv. Mar. Biol. 7: 1-454.

McGowan, J. y P. Walker. 1985. Dominance and diversity maintenance in an oceanic ecosystem. Ecol. Monogr., 55(1): 103-118.

Miller, J. 1974. Nearshore distribution of hawaiian marine fish larvae: Effects of water quality, turbidity and currents. In: The early life history of fish. J. Blaxter. Ed. Springer-Verlag, Berlin, pp. 218-231

Moraga, J. y J. Olivares. 1996. Hidrografía costera de Isla de Pascua, mayo 1994. Cienc. Tecnol. Mar., 19: 47-56.

Mujica, A. 1993. Zooplancton en las aguas que circundan a Isla de Pascua. Cienc. Tecnol. Mar., 16: 55-61.

Olivares, J. y J. Moraga. 1993. Contribución a la descripción de las condiciones oceanográficas del área costera de Isla de Pascua. Cienc. Tecnol. Mar., 16: $47-54$.

Omori, M. y T. Ikeda. 1984. Methods in marine zooplankton ecology. John Wiley \& Sons, New York, 325 pp.

Palma, S. 1985. Plancton marino de las aguas circundantes al archipiélago de Juan Fernández. En: P. Arana (ed). Investigaciones marinas en el Archipiélago de Juan Fernández. Escuela de Ciencias del Mar, Valparaíso, pp. 59-69.

Ponomareva, L. 1963. Euphausiids of the North Pacific, their distribution and ecology. Dokl. Akad. Nauk SSR, 142 pp.

Ramírez, F. 1971. Eufáusidos de algunos sectores del Atlántico Sudoccidental. Physis, 30 (81): 385-405.

Rojas, O. 1980. Determinación de la distribución geográfica y abundancia relativa de huevos y larvas de Túnidos en el Pacífico Sur Oriental. Inst. Fom. Pesq. Chile. AP-80 07. 35 pp.

Silva, N. 1992. Condiciones oceanográficas alrededor de Isla de Pascua durante la primavera de 1979. Cien. Tecnol. Mar, 15: 21-30. 
Simpson, J. y P. Tett. 1986. Island stirring effects on phytoplankton growth. En: J. Bowman, M. Yentsch and W. Peterson (eds.). Lectures notes on coastal and estuarine studies: Tidal mixing and plankton dynamics. New York, pp. 41-76.

Townsed, D., J. Graham y D. Stevenson. 1986. Dynamics of larval herring (Clupea harengus L.) production in tidally mixed waters of the eastern coastal Gulf of Maine. In: J. Bowman, M. Yentsch and W. Peterson (eds.). Lectures notes on coastal and estuarine studies: tidal mixing and plankton dynamics. New York, pp. 253-277.
Watkins, J., D. Morris y C. Riketts. 1985. Nocturnal changes in the mean length of a euphausiid population: vertical migration, net avoidance, or experimental error? Mar. Biol., 86: 123-127.

Youngbluth, M. 1976. Vertical distribution and diel migration of euphausiids in the central region of the California Current. Fish. Bull., 74 (4): 925-936. 